\title{
开放式结构拓扑优化软件设计与研发
}

\author{
梅 帅 陈榦松 张 盛 李云鹏 \\ (大连理工大学工业装备结构分析国家重点实验室 大连 116024)
}

\begin{abstract}
摘要: 随着结构拓扑优化理论的成熟及其在工程中的广泛应用, 大多数商用 CAE 软件都相继开发了结构拓扑优化模块。为 了开发一款既能满足工程方面的实用性需求, 又能满足科研方面的开放性需求的拓扑优化系统。基于具有良好开放性和灵活 扩展性的 SiPESC 平台, 采用面向对象的软件设计思想, 提出了软件设计方案, 基于 $\mathrm{C}++$ 语言和插件技术实现了新一代拓扑 优化系统 SiPESC.TOPO。目前该软件系统已经实现了结构最大刚度设计、结构基频最大化设计、结构最优散热设计等功能, 并成功应用于多个工程问题。
\end{abstract}

关键词: SiPESC.TOPO; 拓扑优化; 结构设计; CAE 软件

中图分类号: $\mathrm{O} 245$; TB115

\section{Design and Development of Open Style Software System for Structural Topology Optimization}

\author{
MEI Shuai CHEN Biaosong ZHANG Sheng LI Yunpeng \\ (State Key Laboratory of Structural Analysis for Industrial Equipment, Dalian University of Technology,
}

Dalian 116024)

\begin{abstract}
With the maturity of the structural topology optimization theory and its extensive application in engineering, structural topology optimization modules have been successively integrated into various commercial CAE software. A structural topology optimization system is developed that not only meet the practical needs for engineering applications, but also meet the open style software demands for scientific research. Based on SiPESC that has good open-ability and extendibility, software framework by object-oriented software design theory is designed, and then developes SiPESC.TOPO. This software system has a variety of functions, such as the maximum stiffness design, the frequency maximization of dynamic structures and the optimum heat dissipation design, and successfully solved many engineering problems.
\end{abstract}

Key words: SiPESC.TOPO; topology optimization; structure design; CAE software

\section{0 前言}

作为结构优化概念设计层次的拓扑优化，能从 根本上定义结构的形式, 它是真正意义上的最优设 计 $^{[1]}$ 。1988 年, BENDSØE 等基于 “微结构” 思想 提出了均匀化方法, 由此, 拓扑优化进入了一个繁 荣时期。随后发展的变密度法, 使得拓扑优化的工 程应用成为可能 ${ }^{[2]}$ 。

随着结构拓扑优化方法的发展及其在工程中 的成功应用, 很多商用 $\mathrm{CAE}$ 软件都增加了结构拓扑 优化功能。SiPESC ${ }^{[3]}$ 是由大连理工大学工业装备结

* 国家自然科学基金(11372064, 91315302)和高等学校学科创新引智计 划(B14013)资助项目。20140606 收到初稿, 20141201 收到修改稿
构分析国家重点实验室研发的面向工程与科学计算 的集成软件系统, 随着 $\operatorname{SiPESC} \mathrm{FEMS}^{[4]}$ (开放式结构 有限元分析系统)功能的完善, 牛飞等 ${ }^{[5]}$ 依托 SiPESC 平台对扩展结构拓扑优化设计功能进行了探索并取 得了良好的效果。

随着拓扑优化系统 SiPESC.TOPO 功能的拓展、 算法的增加, 其可扩展性面临了新的挑战。本文进 一步采用了程序设计模式中的工厂模式 ${ }^{[6]}$ 和 “算法+ 模型” 的软件设计方案 ${ }^{[3]}$, 通过功能模块重组, 对 SiPESC.TOPO 的设计框架进行了调整和改进; 同 时, 将 SiPESC.TOPO 和 SiPESC 平台的集成优化计 算软件系统 SiPESC.OPT 相集成, 实现了通用优化 算法在拓扑优化中的灵活应用, 并且提供了自主扩 展新优化算法灵活机制。目前 SiPESC.TOPO 能够 
解决静力、动力、热传导等多方面的拓扑优化设计 问题, 能够自定义拓扑插值方法、过滤算法等, 能 够灵活替换结构分析模块。

本文主要包含以下五部分内容: 首先概述了 SiPESC 软件系统在结构拓扑优化领域的探索; 第二 部分对软件开发的需求分析进行了详细阐述; 第三 部分根据需求分析结果对软件开发方案进行设计; 第四部分根据方案设计实现基本软件模块; 最后通 过算例验证了拓扑优化系统解决具体问题的正 确性。

\section{1 需求分析}

针对结构拓扑优化的基础研究, 软件系统的开 放性是最迫切的需求, 系统应提供功能灵活扩展的 接口和机制, 从而为研究人员针对算法和功能扩展 留有充分的空间去思考、去拓展; 以 $\mathrm{CAE}$ 软件为例, 用户可以根据自身的需求在软件平台上进行二次开 发, 并且可以在平台上添加新功能, 用户具有相当 大的自由发挥空间。平台具有开放性的同时, 也需 要考虑支持多人多组织协同开发的机制, 以实现研 发工作的协同创新。

结构拓扑优化属于优化设计的初始阶段, 这使 得拓扑优化问题具有复杂性和多样性。在具体问题 的求解过程中, 用户可能需要不同的拓扑优化插值 模型(均匀化法、变密度法等)、不同的结构响应及 灵敏度分析(位移、应力、频率等)、不同的数值问 题处理方法(灵敏度过滤技术、密度过滤技术、周长 约束方法等)。

结构拓扑优化的求解流程可划分为: 结构拓扑 更新、结构响应分析、优化问题建立、优化问题求 解以及优化过程数值问题处理等五个主要部分 ${ }^{[5]}$ 。 由于五部分之间功能相对独立, 且分别有各自的算 法与数据, 研究将根据面向对象软件设计思想, 针 对具体问题提出软件模块设计解决方案。

考虑拓扑插值方法，其实现时存在算法模块与 有限元模型数据交流频繁问题; 但从算法列式上看, 算法模块与具体数据模型的存贮格式无关。可以通 过标准的软件/模块接口将算法与数据分离, 从而实 现算法的通用性, 即算法模块可基于标准接口与其 他有限元模型数据对接。

另一方面对于结构响应及灵敏度分析, 其计算 过程与有限元模型数据格式(结构分析结果)紧密相 关, 为保证复杂大规模问题的计算效率需要针对性 地实现该模块。

根据上述分析，本文将与拓扑插值类似的模块
称为算法类模块, 将与结构响应及灵敏度分析类似 的模块称为任务类模块。对于算法部分，程序要求 算法与数据能够实现动态匹配, 同一算法可以对接 不同数据, 同一数据也可采用不同算法求解; 同时 程序亦要求算法能够动态替换。对于任务部分，程 序要求算法和数据的整体能够动态替换。

这就要求优化程序具有很强的灵活性, 能够根 据所解决的实际问题，快速地将不同算法进行动态 组合; 同时也要求优化程序具有良好的开放性, 能 够允许用户根据自身需求进行算法拓展。以上两点 就是本文工作的出发点和拓扑优化程序在软件层面 所要实现的主要目标。

插件设计是基于动态链接库的软件设计，基于 “平台/微核心十插件” 设计思想的 SiPESC 平台要 求开发者将应用功能按标准接口独立设计为插件, 该思路可类比机械标准件/零部件, 即通过组装插件 得到所需功能。此时的功能模块具有可重用、可维 护、可扩展、动态加载/卸载、使用灵活等优点。

SiPESC 的开放性设计框架解决了结构拓扑优 化问题复杂性和多样性的需求, 使得 SiPESC.TOPO 具备了各部分求解算法的动态选择、替换能力。

\section{2 方案设计}

\section{1 面向对象设计方法}

面向对象的程序设计方法是软件工程一项革 命性的思想和技术，其应用封装、多态、继承技术 进行软件设计, 可避免传统结构化编程的诸多问题。 面向对象设计方法的应用关键是要对问题本身有充 分的认识, 并切实结合软件可维护可扩展的需求, 才有可能设计具备良好性能的软件; 否则简单照搬 面向对象概念，将造成技术滥用，于事无补。针对 结构拓扑优化计算问题, 以固体各向同性惩罚微结 构模型 (Solid isotropic material with penalization, SIMP)式(1)和改进 SIMP 模型式(2)为例介绍面向对 象设计方法

$$
\begin{gathered}
E(\rho)=\rho^{p} E_{0} \quad p \geqslant 1 \\
0<\rho_{\min } \leqslant \rho \leqslant 1 \\
E(\rho)=E^{\min }+\rho^{p}\left(E_{0}-E^{\min }\right) \quad p \geqslant 1 \\
0 \leqslant \rho \leqslant 1
\end{gathered}
$$

式中 $E$ 一一弹性模量;

$E_{0}$ —实际材料弹性模量;

$p$ 一惩罚因子;

$\rho$ 一一材料相对密度。

为避免刚度矩阵奇异, 空洞部分材料密度值取 
为 $\rho_{\min }$, 弹性模量取为 $E^{\min }$ 。

假定公式只计算一个单元，即均按标量考虑， 函数 $E(\rho)$ 代表算法, 变量 $\rho$ 代表数据。函数 $E(\rho)$ 将 算法与数据封装, 并设计接口方法以计算 $E$, 形成 了 SIMP 类。若用户想使用改进 SIMP 模型, 则可 将改进 SIMP 模型的类继承 SIMP 类, 继承计算 $E$ 的接口, 具体采用改进 SIMP 模型计算 $E$ 。当用户 进行优化分析时, 可利用面向对象的多态特性, 实 现动态调用 SIMP 模型或改进 SIMP 模型。

\section{2 软件设计的工厂模式}

软件设计的工厂模式的意图: 定义一个用于创 建对象的公共接口, 让工厂对象根据需要决定实例 化哪一个类 ${ }^{[6]}$; 它使一个类的实例化具备灵活性, 可动态更新与替换(图 1)。工厂模式设计时需要定义 标准接口, 可采用继承技术。如：父工厂类 “扩展 工厂”负责声明创建对象的公共接口 “创建扩 展” ，而子工厂类 “具体扩展工厂” 则负责创建类 实例 “具体扩展”。在此基础上 SiPESC 增加工厂 管理类 “工厂管理器”, 通过算法类型变量动态创 建 “具体扩展工厂”, 实现具体工厂的动态调用, 使程序系统具有良好的灵活性、低耦合性和扩展性。 在结构拓扑优化中, 有多种类型过滤算法供用户选 择。用户可根据问题需要灵活选用算法, 或者根据 实际需求对已有算法进行修正或替换, 或者将算法 的最新研究成果添加到程序中, 在软件设计中可以 采用工厂模式。即用户设定选用算法类型, 通过工 厂管理类的对象确定工厂对象, 从而创建具体算法 对象。工厂管理对象还提供了优先级的动态管理方 式, 针对同一算法类型可依据优先级高低动态返回 具体工厂，实现算法功能的动态替换。

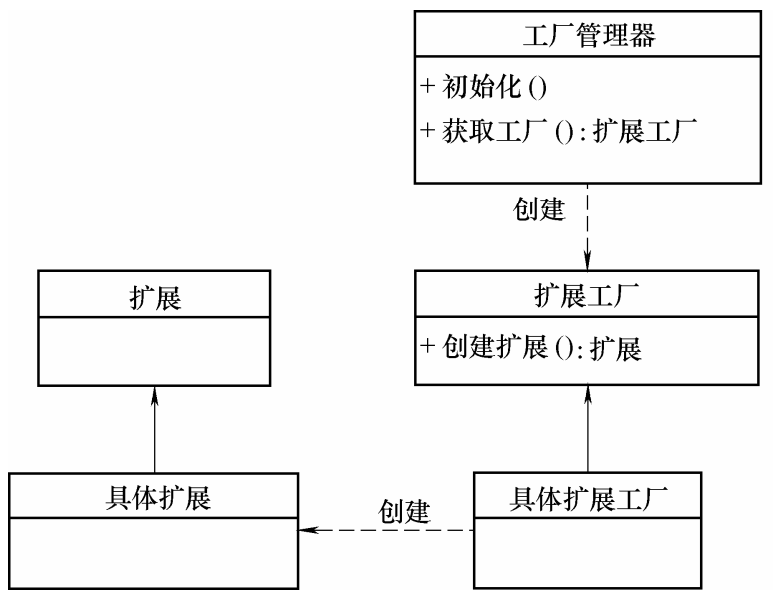

图 1 工厂模式类图

\section{3 “算法十模式” 设计方案}

在通用优化软件系统 ${ }^{[7]}$ 设计过程中, 根据封装 变化和面向接口编程的原则将优化任务分为两部
分：优化算法和优化模型。两部分各司其职, 各自 负责其内部的变化, 利于对优化算法和优化模型(问 题)的扩展。之后, SiPESC 将其发展为通用算法库 设计方案：基于接口的 “算法+模式” 设计方案 (图 2)。“求解器类” 属于算法, 自 “参数类” 中获 取算法参数, 并通过 “模型类” 的统一接口对数据 进行操作, 从而实现整个算法过程。

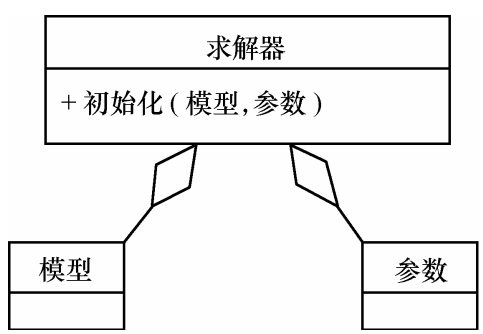

图 2 通用算法库设计

该设计方案符合本文的需求分析, 能够满足算 法与模型可实现动态匹配的要求。通过统一接口可 实现同一算法求解不同的数值模型，也可实现不同 的算法对同一数值模型的求解; 基于统一接口, 可 分别独立地进行算法和模型的编程与调试。

基于 “算法+模式” 的思想, 本文针对拓扑插 值模型及过滤算法等问题提出了具体的可重用软件 实现方案, 下面仍以 SIMP 模型为例进行介绍 (图 3a): 当数据 $\rho$ 代表相对密度时, 拓扑优化模型 为大家熟知的 SIMP 模型; 当以板厚度为设计变量 时, 结构化程序可能需要重新编写程序来实现该功 能, 但经过公式推导, 以板厚为设计变量的变厚度 法与变密度法中的 SIMP 插值模型类似(SIMP 惩罚 因子 $p$ 取定值 3)，此时我们可以直接调用已实现的 $E(\rho)$, 而不必重复类似工作; 同样, 以杆截面积为 设计变量的程序设计也很容易实现。最后, 以上体、 板、杆三种模型均可对接改进 SIMP 模型。

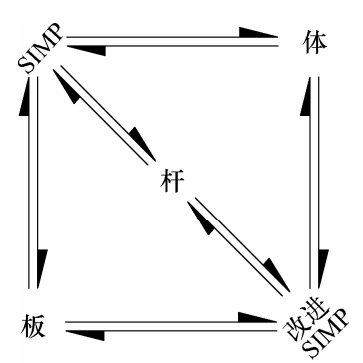

(a)

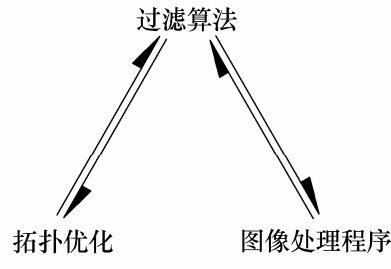

(b)
图 3 “算法+模式” 设计方案

上述算法仅仅是该设计方案在结构拓扑优化 中的应用, 由于该方案中的算法具有通用性, 因此 可以将算法对接不同的模型来解决不同的问题。当 外部程序要使用已经实现的算法时,如图 $3 b$ 中的某 图形处理程序需采用过滤函数处理图像时, 只需要 
加载过滤函数插件并根据算法接口重新实现数据模 型部分即可, 即同一个过滤算法模块, 基于统一接 口可同时应用于结构拓扑优化, 以及图形处理; 该 设计方案可大大节省工作量和提高软件的重用率, 最终节省软件开发成本。

\section{4 集成 SiPESC.OPT}

随着 SiPESC.OPT 功能的日渐完善, SiPESC.TOPO 采用 SiPESC.OPT 作为优化求解器, 即在 SiPESC.OPT 已有功能的基础上对其进行扩 展。由于拓扑优化符合优化基本列式, 因此只需要 对 SiPESC.OPT 的优化模型进行扩展, 以建立新的 优化模型来实现拓扑优化功能(图 4)。“拓扑模型” 继承于 SiPESC.OPT 的优化模型类并实现其接口, 将与结构拓扑优化相关, 而与优化算法无关的功能 (如拓扑插值、正则化等) 置于拓扑模型中实现, 这 样便可以建立通用、可扩展的软件框架方案, 达到 将结构拓扑优化集成到 SiPESC.OPT 中的目的。

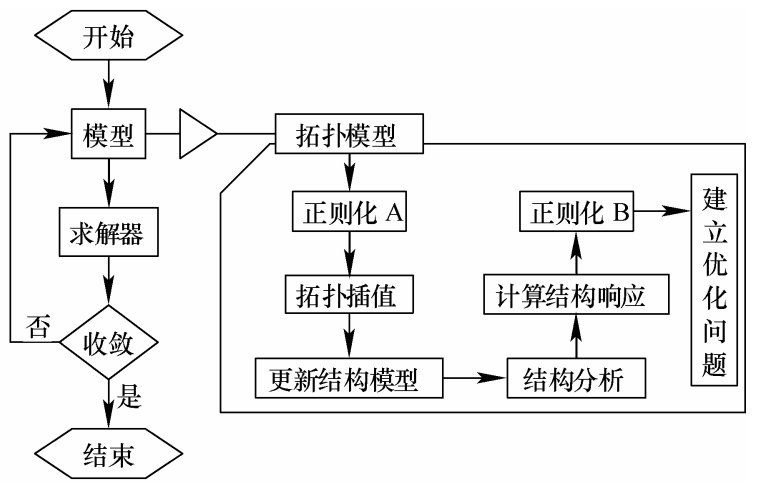

图 4 优化流程图

本文的工作重点是图 4 右侧 “拓扑模型” 中的 拓扑优化计算的相关模块, 优化求解器部分由 SiPESC.OPT 完成。其优点为: (1) 集成了 SiPESC.OPT 丰富的算法库; (2) 简化任务, 优化任 务完全由 SiPESC.OPT 完成; (3) 模块化程序, 便于 二次开发; (4) 插件体系结构, 便于算法动态替换。

\section{5 子模块划分}

如图 4 的 “拓扑模型” 所示, 根据拓扑优化算 法流程将拓扑优化模型划分为六部分：正则化 $\mathrm{A}$, 本文特指由密度类过滤函数处理设计变量, (灵敏度 类过滤自动跳过此步); 拓扑插值, 根据插值模型更 新结构拓扑变量; 更新结构模型, 根据插值结果修 改结构参数; 结构分析, 根据给定条件对结构进行 相应的分析, 此部分集成 SiPESC.FEMS; 计算结构 响应, 依据结构分析结果计算优化列示的指标函数 的响应值及灵敏度; 正则化 $\mathrm{B}$, 对于密度类过滤函 数, 由于结构分析结果与正则化后的设计变量相关, 使得结构响应及灵敏度与正则化后设计变量相关,
因此在建立优化问题前需要将结构响应及灵敏度转 化为正则化前的设计变量的函数, 正则化 $\mathrm{B}$ 与正则 化 A 属于同一模块(对于灵敏度类过滤，该部分由灵 敏度过滤函数处理目标函数与约束函数的灵敏度); 建立优化问题，利用响应值动态组合一个优化问题。

上述六部分经优化处理后, 并考虑参数读入、 结果输出两个方面, 可将拓扑优化模块分为以下七 个子模块(图 5): 参数处理(参数解析); 拓扑结果输 出; 拓扑优化模型; 拓扑插值方案; 结构拓扑更新(更 新有限元模型); 结构响应分析; 优化过程数值问题 处理(过滤策略)。其中, 拓扑插值方案由结构拓扑 更新和结构响应分析两模块共享调用; 结构拓扑更 新、结构响应分析和优化过程数值问题处理属于拓 扑优化模型的子模块; 参数处理和拓扑结果输出属 于依赖数据库的独立模块。基于 SiPESC 插件技术， 并综合应用了工厂模式、“算法十模式” 等软件技 术设计每个模块, 各个模块通过工程数据库进行数 据交换。

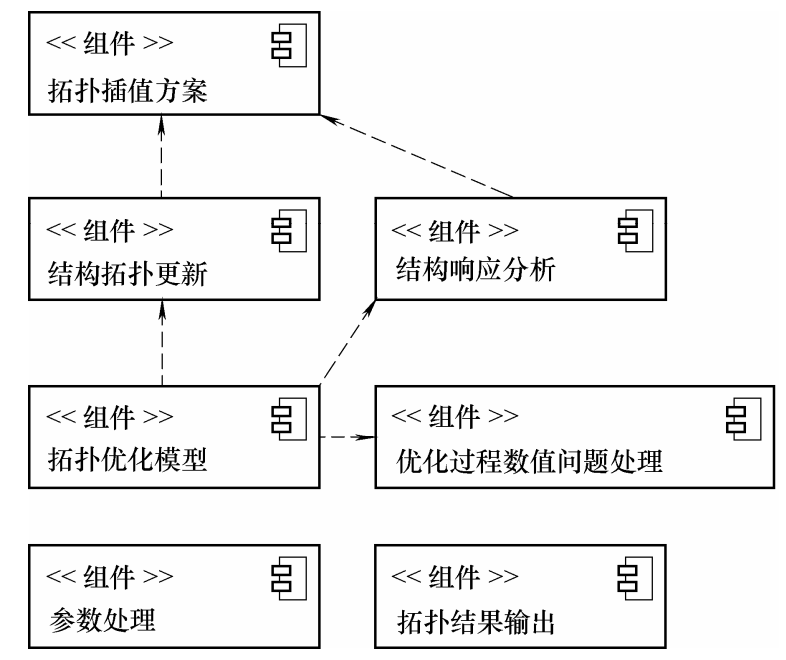

图 5 拓扑优化框架组件图

\section{3 方案实现}

根据第 2.5 节分析, 将拓扑优化分为七个子模 块。其中，总控模块为拓扑优化模型；算法类模块 包括：插值方案和过滤策略；任务类模块包括：结 构拓扑更新、结构响应分析、参数处理和拓扑结果 输出。另外, 为了配合 SiPESC.FEMS, 方便用户使 用 SiPESC 工程数据库, 特别又添加有限元工具 模块。

\section{1 算法类模块}

拓扑插值方案即有限元模型中某变量的更新 方案。目前该模块包含 SIMP 法、改进 SIMP 法、 改进 RAMP 法以及多项式 SIMP 法四种拓扑插值方 
案。为实现算法的通用性, 拓扑插值方案采用了第 2.3 节基于接口的 “算法+模式” 方案进行设计，模 块类图见图 6。其中, 扩展 “SiPESC 插值模型”、

“SiPESC 插值算法” 是与 SiPESC 工程数据库相关 的子扩展, 它们将 SiPESC 数据转换为算法识别的

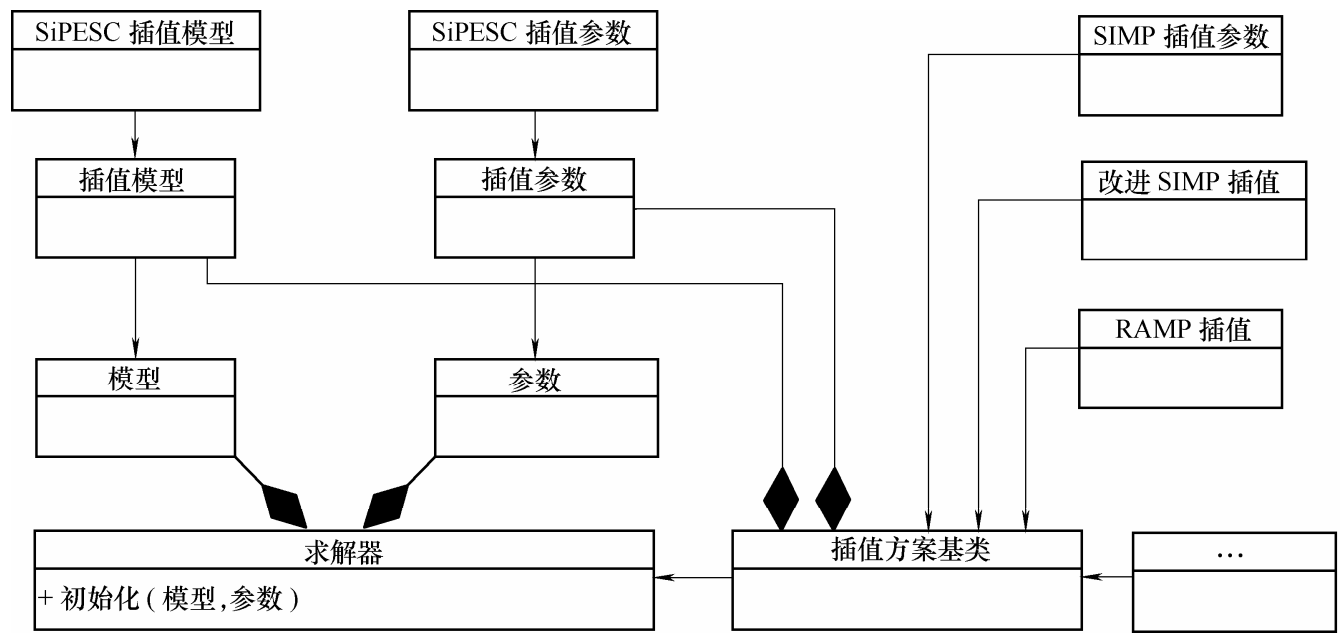

图 6 拓扑插值方案类图
数据类型, 因此当添加一个新算法时, 只需要按照 规定的数据类型编写，而不必考虑将来数据类型的 变化。当采用新数据存储类型时, 只需要重新实现 两个相应的 “模型” 与 “参数” 扩展即可。以上是 算法通用性的实现，下面讨论算法灵活性的实现。
采用第 2.2 节的工厂模式, 可实现拓扑插值方 案的动态替换, 且调用过程简单。模块类图如图 7 所示, 首先实现具体算法以及该算法对应的工厂 (如

“RAMP 插值”与 “RAMP 插值工厂”), 然后由工 厂管理器返回子工厂对象来初始化用户可见的父工 厂对象, 再由子工厂对象返回具体子产品来初始化 用户可见的父产品对象。此时, 用户便得到了特定 的实例化对象(算法), 以此进行相应的分析求解。 工厂管理器有两个接口: 以工厂分类名 (topo.factory.method) 为参数的 “初始化” 接口和以 工厂名(SIMP、RAMP 等)为参数的 “获取工厂” 接 口。用户只需要提供工厂名即可得到所需算法; 若 用户使用自定义算法，则只要根据工厂分类名实现 算法与工厂即可。

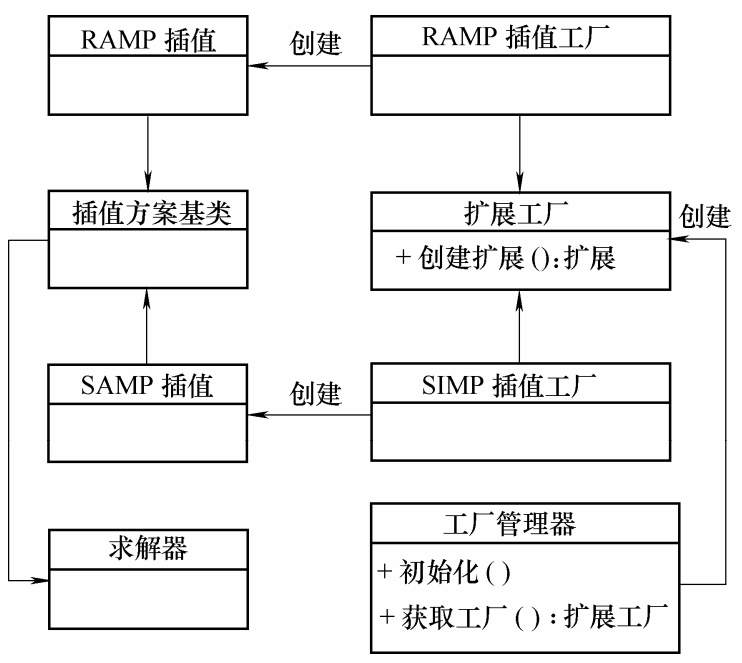

图 7 拓扑插值方案工厂类图
图 6 和图 7 均是面向接口编程的系统架构, 每 个层次仅向上层暴露接口, 而不暴露实现细节。上 层通过接口获取服务, 即上层对于下层仅仅依赖接 口而不依赖具体类。如模块 “结构拓扑更新” 仅仅 依赖拓扑插值方案模块 “插值方案基类” 的接口, 而不依赖 “SIMP 插值” 等具体类, 新增插值方案 “改进 RAMP 插值” 对 “结构拓扑更新” 无任何 影响。该架构具有灵活性与可维护性, 系统设计人 员不必关心各个对象内部是如何实现的，而只需要 了解各个对象之间的协作关系。

同样，过滤策略是优化数值问题处理的一种方 法。目前该模块包含灵敏度过滤、线性密度过滤、 Heaviside 函数非线性密度过滤和体积守恒型 Heaviside 函数非线性密度过滤等。过滤策略作为算 法类模块的一个, 同样采用上述实现方法, 在此不 再赘述。

\section{2 任务类模块}

结构拓扑更新, 即根据拓扑插值方案更新有限 元模型中相应的变量, 如静力拓扑优化问题需更新 单元刚度阵、动力拓扑优化问题需要更新单元刚度 阵和单元质量阵。

任务类模块中的结构拓扑更新包括前述的静 力问题、动力问题，以后还可能包含热传导问题、 机构问题等，这就要求开发者采用第 2.2 节的工厂 模式实现此模块, 以实现算法的动态调用。由于此 部分只采用了工厂模式，因此较算法部分简单，只 需要添加任务(产品)与工厂两个扩展并重新实现接 
口即可, 与第 3.1 节的后半部分类似。

结构响应与灵敏度分析(应变能、节点位移、结 构基频等)、参数解析(优化参数卡片、拓扑插值参 数卡片等)和拓扑结果输出(拓扑结果、含位移拓扑 结果、含应力拓扑结果等)的软件层面的本质与结构 拓扑更新均为多问题型任务, 因此实现方法与前述 基本相同，不再赘述。

以上任务类及第 3.1 节的算法类均是在工程数 据库基础上实现的, 数据库的开放性便于用户自数 据库中存取结构分析数据、优化参数及优化结果。 如计算应变能的单元刚度阵与单元位移, 计算结构 基频灵敏度的单元特征矢量等。使用前首先必须以 数据库初始化模块, 下面以示意代码说明程序实现 的便捷性。

initialize(m)

MDataManager rV,eCM;

rV.open $(m, .$.$) ;$

eCM.open $(\mathrm{m}, \ldots)$;

MVector dV;

MVectorData data $=$ rV.getDataAt $(\ldots)$;

data $>\mathrm{dV}$;

MElementControlMatrixData eCMD = eCM.get

Data (eleId);

MVector $\mathrm{eDV}=\mathrm{cT}$.getElementControlResult (eCMD,dV, MVector());

第 1 行, 以某级数据库目录 m(MDataModel 类 型)初始化模块; 第 $2 \sim 4$ 行, 声明并打开数据对象 管理器: 结果位移矢量 $\mathrm{rV}$ 、单元控制阵 $\mathrm{eCM}$; 第 5 7 行, 自结果位移矢量管理器中取得对应工况的位 移矢量 data, 并转换为 MVector 类型 $\mathrm{dV}$; 第 8 行, 自单元控制阵管理器中取得 eleId 号单元所对应的 单元控制阵 eCMD; 第 10 行, 由控制阵转换扩展 $\mathrm{cT}$ 将整体位移矢量转换为单元位移矢量 $\mathrm{eDV}$ 。

\section{3 总控模块}

拓扑模型作为 SiPESC.TOPO 的总控模块、 SiPESC.OPT 的子模块, 在 SiPESC.OPT 的可扩展软 件框架下实现了拓扑优化算法类模块和任务类模块 的整合，以及不同算法、任务的动态调用。

目前整个拓扑优化程序是基于 SiPESC 工程数 据库进行数据存储和交换的。如图 5 所示, “参数 处理” 将参数数据存入数据库, 而 “拓扑结果输 出” 自数据库中获取数据并生成后处理文件, 其他 几个模块均由 “拓扑优化模型” 支配。

此部分的设计思想依然是工厂模式, 首先根据 各个模块的类型初始化工厂管理器(如优化问题类 型的选择, 即结构拓扑更新分类 topo.factory.fems),
然后自数据库中获取与算法、任务对应的工厂名并 由工厂管理器创建工厂对象，最后由工厂对象返回 具体的算法、任务对象。将创建的算法、任务对象 进行有机组合, 最终形成拓扑优化系统。优化问题 类型选择的插件使用方法如下。

MExtensionManager eM = MExtensionManager:: getManager();

MExtensionFactoryManager $\mathrm{fM}=$ eM.create Extension("org.sipesc.utilities.MExtensionFactoryMa nager");

fM.initialize("topo.factory. fems");

MExtensionFactory $\mathrm{f}=\mathrm{fM}$.getFactory ("Static Topology");

MTopologyProblem ts = f.createExtension();

第 1 行, 获取全局插件管理器 $\mathrm{eM}$; 第 3 行, 使用插件管理器创建工厂管理器扩展 $\mathrm{fM}$; 第 6 行, 初始化工厂管理器; 第 7 行，工厂管理器根据工厂 名创建静力问题拓扑优化工厂 $\mathrm{f}$; 第 9 行, 由工厂 创建产品 ts。

若将代码中的 StaticTopology 替换为 Thermal Topology, 并加载温度场相关插件(第 3.4 节), 此时 程序便切换到温度场拓扑优化。对于微机电系统、 声场、电磁场等问题领域的切换也是如此便捷。

\section{4 模块扩展}

用拓扑优化方法来对结构的最佳散热拓扑形 式进行设计是一种新的尝试。其方法是：以最小化 结构的散热弱度(热量传递势容耗散函数)为目标函 数, 在一定约束下通过优化计算得到材料在最佳散 热效果情况下拓扑分布。

当需要在 SiPESC 平台上添加一个新功能时, 若属于创新性功能(拓扑优化功能), 则需要根据平 台的现有接口设计新模块; 若属于更新性功能(将结 构问题拓展到热传导问题), 则只需要对现有任务类 模块进行更新，重新实现接口即可。

对于热传导功能的扩展, 本文需要解决以下问 题: (1) 结构分析系统具有温度场的分析功能; (2) 在 “结构拓扑更新” 模块中添加热传导拓扑优化, 即 添加子类实现更新热导率矩阵的方法; (3) 在 “结构 响应分析” 模块中添加子类实现计算散热弱度及其 灵敏度的方法。

对于温度场分析和位移场分析, 温度 $\boldsymbol{T}$ 等价于 位移 $\boldsymbol{U}$, 内部发热量 $\boldsymbol{Q}$ 等价于体力 $\boldsymbol{F}$, 热导率矩阵 $K_{R}$ 等价于结构刚度阵 $\boldsymbol{K}_{S}$, 散热弱度 $\boldsymbol{T}^{\mathrm{T}} \boldsymbol{K}_{R} \boldsymbol{T}$ 等价于 柔顺性 $\boldsymbol{U}^{\mathrm{T}} \boldsymbol{K}_{S} \boldsymbol{U}$, 因此以散热弱度为目标函数的热传 导拓扑优化等价于以柔顺性为目标函数的结构拓扑 优化。

上述变量之间是等价的，若想使用结构柔顺性 
插件解决温度场问题, 利用解决矢量场问题的扩展 解决标量场问题, 可令温度场的数据库存储格式与 位移场分析的数据库存储格式相同。对于 SiPESC, 温度场与位移场分析中的数据路径、数据名称及数 据格式均一致, 满足了上述需求。过滤策略只与单 元(设计变量)相关, 而与分析类型无关; 拓扑插值 模型与系数矩阵 (刚度阵、热导率矩阵)相关; 指标 函数(响应)与结构分析结果相关。本文直接利用结 构拓扑优化中的柔顺性扩展解决问题(2)与问题(3)。

虽然散热弱度具有整体效应, 最优点的性态良 好, 但是通过散热弱度描述结构的散热性能只是真 实最优设计(最高温度最低化)的表征和近似, 在某 些情况下存在较大误差。因此学者们提出了几何平 均温度、温度方差等指标作为优化目标。SiPESC 平台良好的开放性和灵活的扩展性可以让用户方便 地添加以上指标函数, 同样只需要在第(3)条 “结构 响应分析” 模块中增加子类实现计算指标函数及其 灵敏度的方法即可。

\section{4 数值算例}

\section{1 空间壳组合结构拓扑优化设计}

如图 8a 所示的槽型梁结构, 长 $l=5 \mathrm{~m}$, 高 $a=0.5$ $\mathrm{m}$, 宽 $b=0.25 \mathrm{~m}$, 壁厚 $0.05 \mathrm{~m}$; 右端下部与距右端 $1 \mathrm{~m}$ 处简支; 结构左端安装一支架, 分别与结构左 端中部和距结构左端 $1 \mathrm{~m}$ 下部铰接, 支架受坚直向 下 $10 \mathrm{kN}$ 的集中力。材料的弹性模量 $E=206 \mathrm{GPa}$, 泊松比 $\nu=0.3$ 。采用 $0.025 \mathrm{~m} \times 0.025 \mathrm{~m}$ 的 4 节点壳单 元对槽型梁结构进行网格离散, 采用杆单元对左端 支架进行离散。设定槽型梁为可设计域, 设计域材 料体分比为 0.3 , 进行最大化结构刚度设计; 采用体 积守恒型非线性密度过滤, 过滤半径 $0.05 \mathrm{~m}$; 优化 求解使用 MMA 算法。

梯度算法 MMA 的求解效率较高, 且对多设计 变量适应性强。硬件环境: Intel(R) Core(TM)2 Duo E6550 $2.33 \mathrm{GHz}$ 、内存主频 $332.5 \mathrm{MHz}$ 及 32 位系统 Ubuntu12.04 下, 8000 个设计变量单步需要 26.129 S。优化后的最优拓扑如图 $8 \mathrm{~b}$ 所示, 最优设计拓扑 构型清晰, 传力路径合理; 目标函数迭代历史曲线 如图 8c 所示, 目标收玫趋势光滑。由于体积守恒非 线性密度过滤中每隔 50 步会加强一次非线性强度， 因此目标收敛曲线每隔 50 步会跳跃一次, 不影响整 体收玫性。此算例验证了 SiPESC.TOPO 在三维空 间壳和组合结构中结构拓扑优化功能的适用性和正 确性。

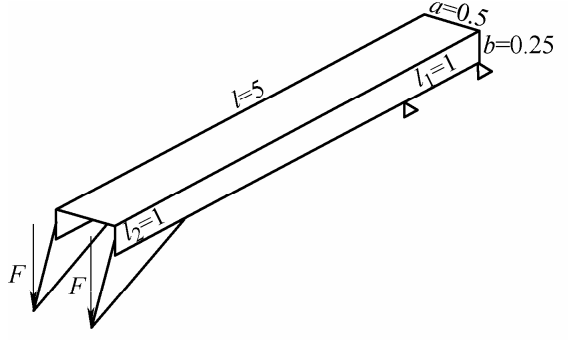

(a) 几何模型 $(m)$

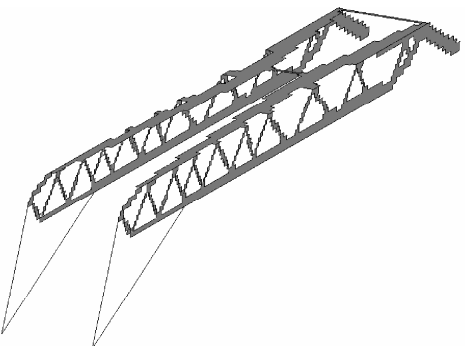

(b) 优化结果

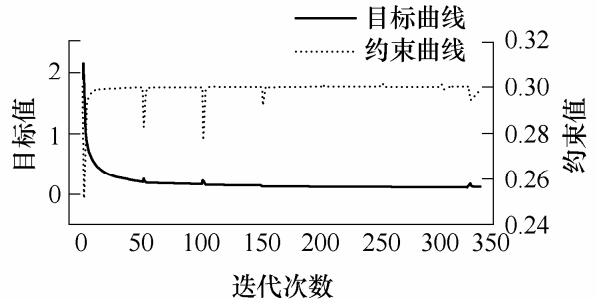

(c) 目标迭代历史

图 8 空间壳组合结构拓扑优化设计

\section{2 热传导拓扑优化设计}

如图 9a 所示的平板导热结构, 面内尺寸为 160 $\mathrm{mm} \times 160 \mathrm{~mm}$, 厚度为 $1 \mathrm{~mm}$; 平板左端中部安装散 热装置, 装置长 $16 \mathrm{~mm}$, 且保持恒定温度 $0{ }^{\circ} \mathrm{C}$, 其 余边界均为绝热, 平板具有均布热源, 其生热速率 为 $1 \times 10^{6} \mathrm{~W} / \mathrm{m}^{3}$; 材料的热导率 $k_{p}=100 \mathrm{~W} /(\mathrm{m} \cdot \mathrm{K})$ 。采 用 4 节点平面单元对结构进行离散, 初始网格划分 为 $160 \times 160$ 。高热导材料体分比为 0.3 , 进行最小化 最高温度设计。采用体积守恒非线性密度过滤, 过 滤半径 $1.8 \mathrm{~mm}$ ，优化算法采用 MMA。

优化后的最优拓扑如图 $9 \mathrm{~b}$ 所示, 由于各个单 元均为热源, 这会导致材料用量为零(低热导率)处 的温度过高; 因此本算例中设置平板的基体厚度为
$0.1 \mathrm{~mm}$, 此时, 优化列式中的约束值实际为 0.37 。 此经典算例验证了 SiPESC.TOPO 在热传导拓扑优 化方面的适用性和正确性。

\section{3 基频最大化拓扑优化设计}

如图 10a 所示的薄板结构, 面内尺寸为 0.15 $\mathrm{m} \times 0.1 \mathrm{~m}$, 厚度为 $0.00122 \mathrm{~m}$, 四边固支。材料的弹 性模量 $E=70.0 \mathrm{GPa}$, 泊松比为 $v=0.3$, 材料密度 $\rho_{0}=$ $2700 \mathrm{~kg} / \mathrm{m}^{3}$ 。给定材料用量上限 0.6 , 在薄板形心处 的集中质量为可用材料总质量的 0.1 , 即 $M_{0}=0.003$ $\mathrm{kg}$ ，对结构进行最大化基频设计。采用 4 节点平面 应力单元对结构进行离散, 初始网格划分为 $100 \times 50$ 。采用体积守恒型非线性密度过滤, 过滤半 径 $0.002 \mathrm{~m}$, 优化求解采用 MMA 算法。 


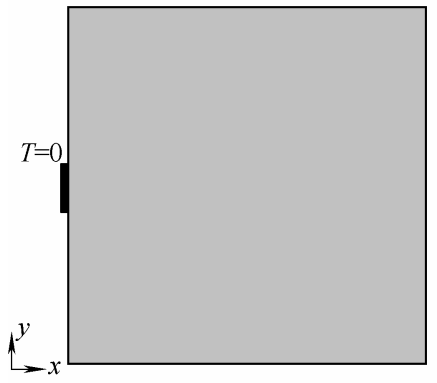

(a) 几何模型

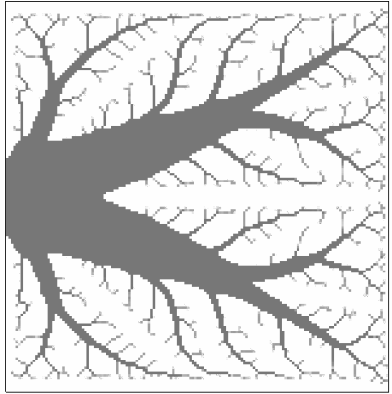

(b) 优化结果

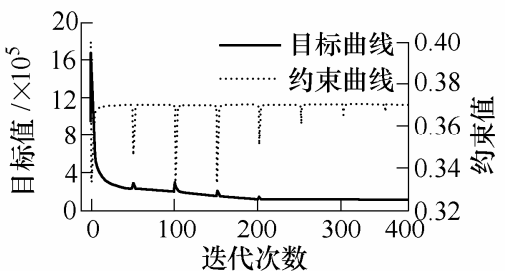

(c) 目标迭代历史

图 9 热传导拓扑优化设计

优化后的最优拓扑如图 $10 \mathrm{~b}$ 所示; 目标函数迭 代历史曲线如图 10c 所示, 目标收玫趋势光滑。此
经典算例验证了 SiPESC.TOPO 在动力拓扑优化方 面的适用性和正确性。

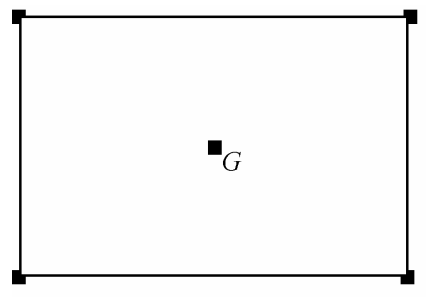

(a) 几何模型

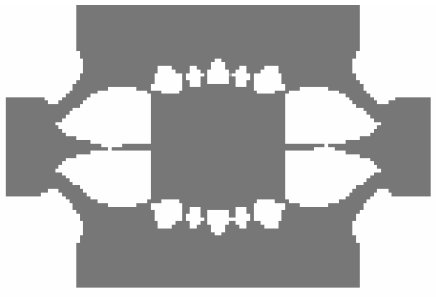

(b) 优化结果

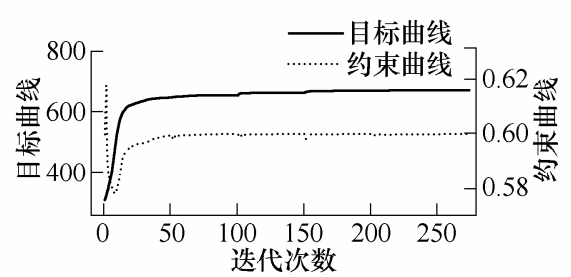

(c) 目标迭代历史

图 10 基频最大化拓扑优化设计

\section{5 结论}

本文基于计算力学软件集成平台 SiPESC, 探讨 了开放式结构拓扑优化软件系统 SiPESC.TOPO 的 设计与开发。在总结前人经验的基础上, 通过面向 对象技术、软件设计模式和 “算法+模式” 设计方 案等一系列措施, 使得 SiPESC.TOPO 具备了强大 的二次开发能力, 并且支持多人多组织协同开发。 平台的开放性使科研人员能够方便地进行科学研 究, 插件机制保证了对个人研究成果的保护, 同时 可以将插件以个人名义发布, 从而将最新研究成果 应用于工程实际。

目前该软件框架已经设计完成，同时实现了基 本的结构拓扑优化功能。由于拓扑优化问题具有复 杂性和多样性, 因此要使 SiPESC.TOPO 具有更强 大的解决问题的能力, 可通过软件的二次开发来继 续丰富软件功能和算法。

\section{参 考 文 献}

[1] BENDSØE M P, SIGMUND O. Topology optimization: Theory, methods, and applications [M]. Berlin: Springer, 2003.

[2] MLEJNEK H P. Some aspects of the genesis of structures $[\mathrm{J}]$. Structural and Multidisciplinary
Optimization, 1992, 5(1): 64-69.

[3] 张洪武, 陈燓松, 李云鹏, 等. 面向集成化 CAE 软件 开发的 SiPESC 研发工作进展 $[\mathrm{J}]$. 计算机辅助工程, 2011, 20(2): 39-49.

ZHANG Hongwu, CHEN Biaosong, LI Yunpeng, et al. Advancement of design and implementation of SiPESC for development of integrated CAE software systems [J]. Computer Aided Engineering， 2011，20(2): 39-49.

[4] 张盛, 杨东生, 尹进, 等. SiPESC.FEMS 的单元计算 模块设计模式 [J]. 计算机辅助工程, 2011, 20(3): 49-54. ZHANG Sheng, YANG Dongsheng, YIN Jin, et al. Design pattern of element computation module of SiPESC.FEMS[J]. Computer Aided Engineering, 2011, 20(3): 49-54.

[5] 牛飞, 梅帅, 张盛, 等. 基于 SiPESC 平台的拓扑优化 模块的开发及应用 $[\mathrm{J}]$. 大连理工大学学报, 2013(1): 162-168.

NIU Fei, MEI Shuai, ZHANG Sheng, et al. Development and application of the topology optimization module based on SiPESC[J]. Journal of Dalian University of Technology, 2013(1): 162-168.

[6] GAMMA E, HELM R, JOHNSON R, et al. Design patterns: Elements of reusable object-oriented software [M]. Addison-Wesley Professional, 1994: 116-117.

[7] 杨春峰, 陈飙松, 张盛, 等. 通用集成优化软件 
SiPESC.OPT 的设计与实现 [J]. 计算机辅助工程, 2011, 20(4): 42-48.

YANG Chunfeng, CHEN Biaosong, ZHANG Sheng, 作者简介: 梅帅, 男, 1986 年出生。主要研究方向为结构优化软件系统。 E-mail: meishuai291@gmail.com et al. Design and implementation of general integrated 陈杀松(通信作者), 男, 1973 年出生, 博士, 教授, 博士研究生导师。 optimization design software SiPESC.OPT[J]. Computer Aided Engineering, 2011, 20(4): 42-48.

主要研究方向为计算力学软件系统、结构与多学科优化。

E-mail: chenbs@dlut.edu.cn

\section{第 4 届上银优秀机械博士论文奖——佳作奖}

\section{综采工作面 “三机” 控制中设备定位及任务协调研究}

作者：樊启高

毕业学校: 中国矿业大学

指导教师: 李威

随着国家能源需求日益旺盛，以煤炭为主的能源结构将持续很长时间，而煤炭资源的安全高效开采问题，已经成为资 源环境领域的重点研究课题。本文在国家 “ 863 ” 课题基金的资助下，以综采工作面 “三机” 装备一采煤机、液压支架和刮 板输送机为研究对象, 以实现井下工作面无人化采矿为目标, 开展 “三机” 设备定位及任务协调研究。通过掌握 “三机” 协 同运动规律, 建立 “三机” 系统运动学模型, 进而综合惯性导航理论以及超宽带无线传感网络定位理论, 构建采煤机 INS/UWB 协同定位模型，实现封闭空间下采煤机高精度定位定姿。在此基础上，分析采煤机、液压支架与刮板输送机相互约束规律， 开展工作面“三机”参数动态匹配技术研究, 构建“三机”约束集合, 进而建立 “三机” 任务协调机制, 实现 “三机” 正常调度 优化以及冲突消解, 为智能化采矿提供理论支撑及技术支持。主要研究工作包括:

(1) 综采工作面 “三机” 系统运动学模型的建立。分析了采煤机工作空间及运动规律, 完成了采煤机姿态及位置表达, 构建了采煤机空间运动学模型。在此基础上, 掌握了液压支架推溜、移架规律, 通过制定液压支架联动规则, 建立了液压支 架跟机联动模型。

(2) 封闭空间下采煤机动态定位策略的研究。首先对纯惯性导航系统下采煤机定位展开了研究, 确定了采煤机惯性导航 系统编排, 构建了采煤机惯性导航方程, 提出了基于四元数的姿态解算策略, 进一步在惯性试验平台上对纯惯性导航下采煤 机定位性能进行了测试, 揭示了惯性导航系统的优势与不足; 在此基础上提出基于 INS/UWB 的采煤机组合导航策略, 综合 两个子系统存在的优势, 研究了位置组合模式下的 INS/UWB 耦合机制, 并进行了组合定位系统的可行性仿真研究, 验证了 理论模型的正确性。

(3) INS/UWB 组合导航数据融合策略的研究。掌握了采煤机工况下 INS/UWB 组合导航系统误差传播特性, 对 INS 与 UWB 子系统误差分别进行了建模, 构建了系统误差方程, 在此基础上确定了 INS/UWB 组合导航系统状态方程与量测方程, 研究了基于模糊自适应 Kalman 滤波技术的 INS/UWB 组合导航数据融合策略, 并进行了 INS/UWB 组合导航仿真研究; 最后 通过构建 INS/UWB 协同定位平台, 开展了采煤机定位定姿试验研究, 验证了 INS/UWB 组合导航理论模型的正确性, 同时 确定了系统精度。

(4) 工作面 “三机” 集合约束下任务协调机制的研究。分析了采煤机、液压支架和刮板输送机之间的约束关联，进而研 究了工作面 “三机” 工作参数动态匹配技术，在此基础上建立了 “三机” 协同任务控制模型，并采用动态规划理论进行了模 型求解，进一步提出了基于通用部分全局规划的 “三机” 任务协调策略，并构建了 “三机” 协同运动仿真平台，开展了工作 面 “三机” 任务协调试验研究, 结果表明, 通过建立 “三机” 实时任务视图, 能够进行实时任务约束检测, 实现 “三机” 协 同工作，同时提高 “三机” 机组设备效率与总体效能。 